\title{
Long-range passive IR sensor
}

\author{
H. Madura, Z. Sikorski, H. Polakowski, M. Kastek \\ Institute of Optoelectronics, Military University of Technology, \\ 2 Kaliskiego Str., 00-908 Warsaw
}

\begin{abstract}
The paper presents application and essential characteristics of IR passive sensors as well as principle of their operation. An analysis method of radiation signals for detected targets (man) has been described. Propriety of selection of longwavelength detection band of $8 \div 12 \mu \mathrm{m}$, in respect with low temperatures contrast of a target and hazard of false alarms resulting from fluctuation of background radiation and reflected sun radiation, has been motivated. Also usefulness of optical filters eliminating radiation at wavelengths shorter than $8 \mu \mathrm{m}$ was given.
\end{abstract}

\section{Introduction}

\subsection{Application and fundamental properties of passive IR sensors}

Passive IR sensors are used in object protection devices due to their specific properties distinguishing them from among other devices used for this purpose $[1,2,3]$. Passive IR sensors can operate both during the day and night. As opposed to the sensors operating within visible and near-infrared radiation they can operate properly in the conditions of limited visibility (e.g., in a fog or smoky area). They detect heat radiation that is inseparable attribute of the material objects. It should be pointed out that passive IR sensors are completely undetectable devices what differ from active systems (e.g., microwave, radio, laser, and optical barriers). They are safe for service personnel, for eyes and environment.

\subsection{Principle of operation of passive IR sensors}

The collecting optics of passive IR sensor is decisive for quality of device operation as well as effects quantity and spectral composition of the radiation focused on a detector. The detector integrates optical signal over its surface and time and next converts it into electrical signal. However, it is simultaneously a source of noise. Electronic systems for signal conversion amplify and filter the signal that is next analysed by automated decisive system. Frequency and noise characteristics of electronic system influence on correctness of final analysis of the signal, the result of which is decision about object detection. For the objects smaller than the field of view of the optical sensor system, the signal-to-noise ratio decreases with square of the distance between object and sensor. It results from the above that design of long-range passive IR detector employing uncooled detectors requires detailed analysis of many optical and electronic problems [4,5].

\section{Analysis of radiation signals from a detected object}

The paper presents an analysis of limits of object detection by means of passive IR sensors. For this purpose, investigations on statistic properties of temperature distributions of objects and background were carried out. 


\subsection{Assumptions for analysis}

As a detected object, the man moving with velocity of $0.3-3 \mathrm{~m} / \mathrm{s}$, at the distance from 50 to $200 \mathrm{~m}$, was taken. The investigations were made using long-wavelength thermovision camera Inframetrics 760. Scope of the research comprises registration of thermograms made in winter and summer conditions. Figure 1 shows one of variants of sensor position and shape (dimensions) of the protected zone.
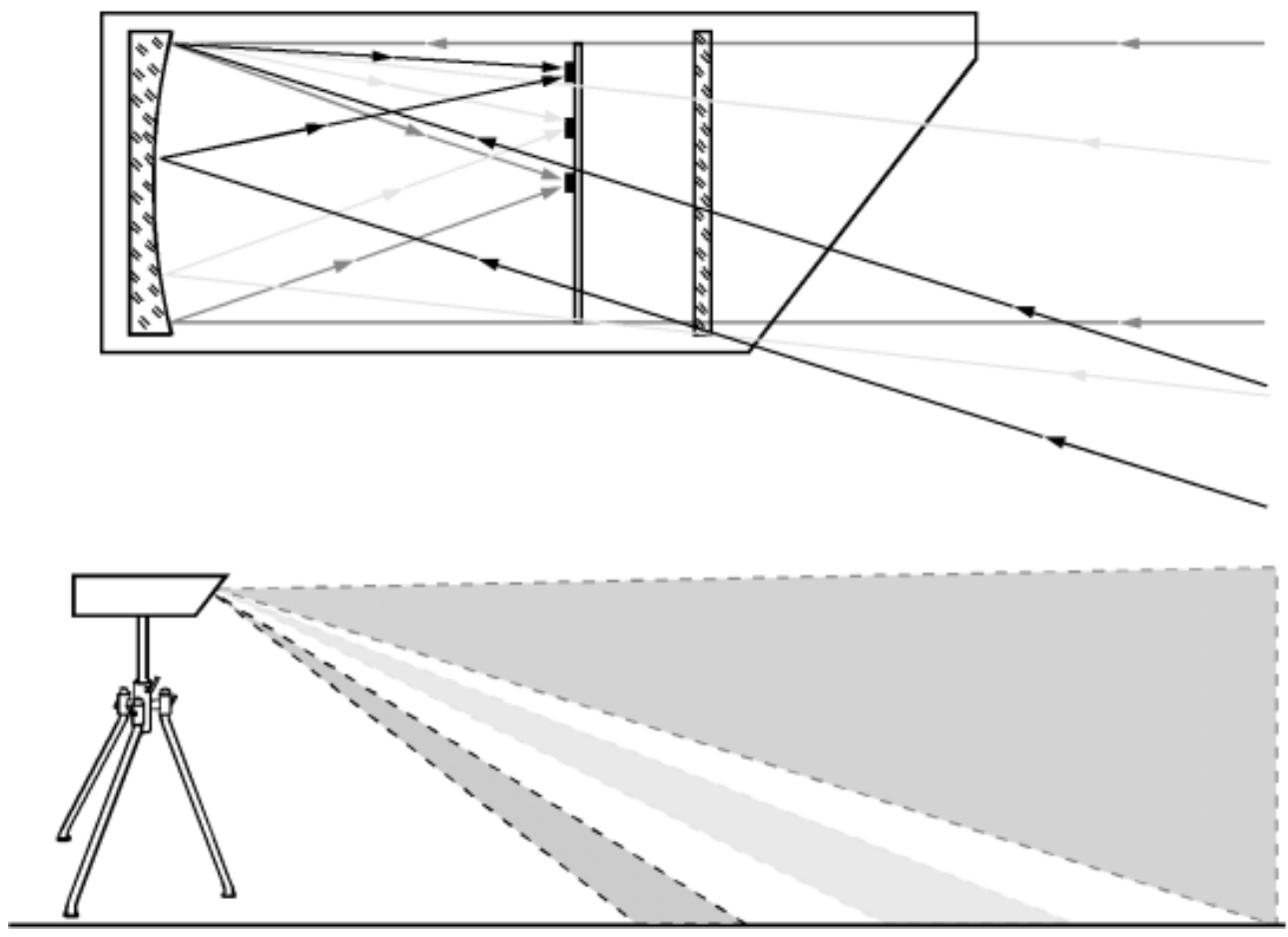

Fig. 1. Protection zone of a passive IR sensor (sensor is fixed at $\sim 2 \mathrm{~m}$ height above he ground)

\subsection{Analysis of radiation signals}

On the basis of the thermograms obtained with Inframetrics 760 camera, the static temperature distributions of people observed at various backgrounds, various conditions (winter and summer), and during day and night were observed [4]. Analysis was carried out for the most difficult conditions for people detection, i.e., conditions of intense solar light in summer (at night and in winter the temperature contrast between a man and background is higher). An exemplary thermogram of a man registered during a summer day is shown in Fig. 2. It illustrates variety of backgrounds that can give either positive or negative temperature contrast. At night and in winter the temperature contrasts are positive.

For further analysis the most difficult case is taken. It is for man seen on a background that is characteristic for summer day conditions. The following average values are assumed; the man temperature $\mathrm{T}_{0}=302 \mathrm{~K}$, the background temperature $\mathrm{T}_{\mathrm{b}}=300 \mathrm{~K}$ and their difference $\Delta \mathrm{T}=\mathrm{T}_{0}-\mathrm{T}_{\mathrm{B}}=2 \mathrm{~K}$.

The thermogram of a man was approximated by an equivalent rectangle of $170 \times 50 \mathrm{~cm}$ (front view) and $170 \times 35 \mathrm{~cm}$ (end view). These dimensions were taken according to the Polish Standard PN-931E-0890126. 


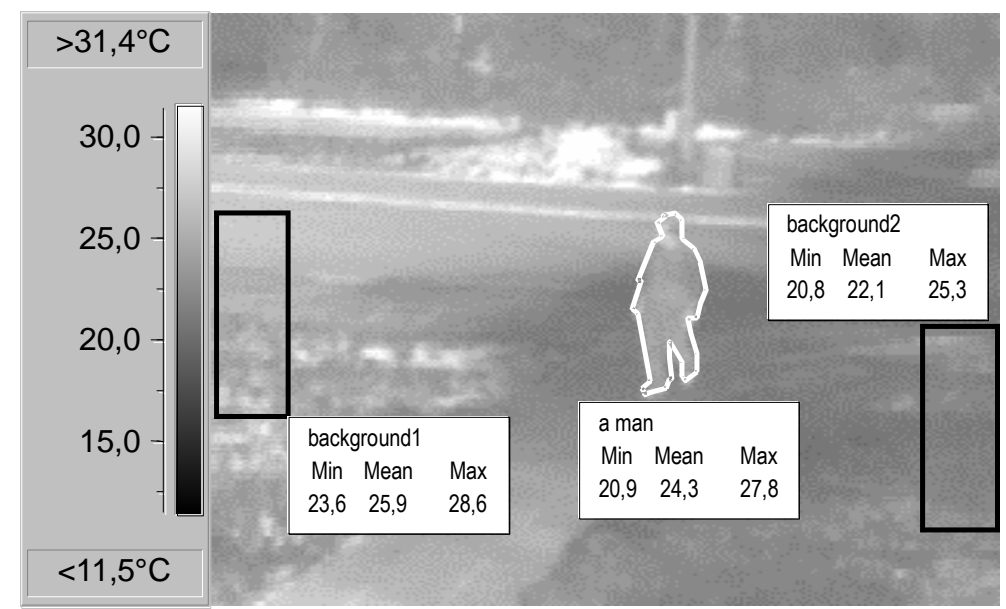

Fig. 2. Exemplary thermogram of a man and his nearest environment obtained for the conditions of sunlit summer. Determined statistic parameters of temperature distributions for the denoted areas are shown in figure.

\subsection{Selection of detector type and spectral range of its operation}

According to the Wien's rule, the maximum of radiation intensity of an object (man) is for the following wavelength

$$
\lambda_{\max }=\frac{2897.82}{T_{0}}=9.6 \mu \mathrm{m},
$$

where $\mathrm{T}_{0}=300 \mathrm{~K}$.

For the given temperature, the maximum sensitivity to a temperature change is for the wavelength $\lambda_{\text {opt }}$, shorter than $\lambda_{\max }$. A function described in Eq. 2 reaches its maximum for the wavelength $\lambda_{\text {opt }}$

$$
\left.\frac{\partial M_{\lambda}(T)}{\partial T}\right|_{T=T_{0}}=\frac{h c \exp (h c / \lambda k T)}{\lambda k T^{2}[\exp (h c / \lambda k T)-1]} M_{\lambda}(T)
$$

where $M_{\lambda}(T)$ is the spectral density of energetic luminous exitance (luminous emittance). The determined optimal wavelength is $\lambda_{\text {opt }}=8 \mu \mathrm{m}$, so it is advantageous to use the sensitive detector of long-wavelength detection range (8-12 $\mu \mathrm{m})$. Denoting increase in the energetic exitance as $\delta \mathrm{M}$, caused by increase in the object temperature the $\delta \mathrm{T}=1 \mathrm{~K}$, we can compare sensitivity for mid- and long-wavelength range of detection. The values described by the formula

$$
\frac{\delta M}{\delta T}=\int_{\lambda_{1}}^{\lambda_{2}} \frac{\delta M_{\lambda}(T)}{\delta T} d T
$$

in both spectral ranges, for $\mathrm{T}=300 \mathrm{~K}$, are

$$
\begin{aligned}
& \left.\frac{\delta M}{\delta T}\right|_{3-5 \mu m}=2.1 \times 10^{-5} \mathrm{~W} \mathrm{~cm}^{-2} \mathrm{~K}, \\
& \left.\frac{\delta M}{\delta T}\right|_{8-12 \mu m}=19.7 \times 10^{-5} \mathrm{~W} \mathrm{~cm}^{-2} \mathrm{~K},
\end{aligned}
$$


and their ratio is equal to

$$
\frac{\left.\frac{\delta M}{\delta T}\right|_{8-12 \mu m}}{\left.\frac{\delta M}{\delta T}\right|_{3-5 \mu m}}=\frac{19.7 \times 10^{-5}}{2.1 \times 10^{-5}}=9.4
$$

The obtained numerical values confirm validity of a choice of longwavelengthe detection band $(8-12 \mu \mathrm{m})$. Selection of long-wavelength band is proper because intensity of the reflected solar radiation, being one of noise components causing false alarms, is significantly lower in this range in comparison with midwavelength $(3-5 \mu \mathrm{m})$ range. Because of low temperature contrast of an object and a possibility of false alarms (as a result of background fluctuation) a pyroelectric lithium tantalate detector of a specific construction, shown in Fig. 3, was applied.

The detector has two active elements of dimensions $2.5 \mathrm{~mm}$ (vertically) $\times 1 \mathrm{~mm}$ (horizontally) separated with vertical slot of a width $1 \mathrm{~mm}$. The detector acts when the difference of the signals from both elements appears. So, it is slightly sensitive to background fluctuations and slow changes in intensity of reflected solar radiation. Moving object that crosses horizontal barrier (determined by the detector's angle of view) first produces the signal in one of the detective elements and next in the other one. The normalised detectivity of this detector is

$$
D^{*}=2.2 \times 10^{8} \mathrm{~cm} \sqrt{\mathrm{Hz}} W^{-1}
$$

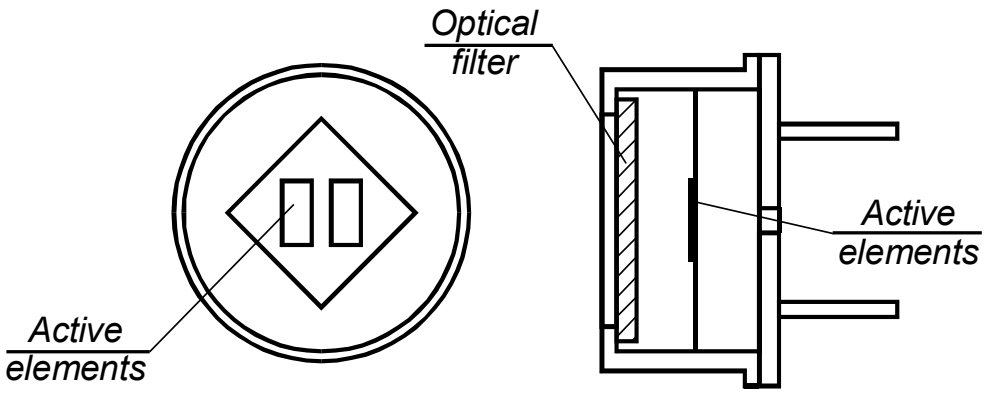

Fig. 3. Construction of two-element pyroelectric detector.

The analyses results were used for elaboration of longrange passive IR sensor within the frame of the grant of the Polish Committee for Scientific Research (grant No. 148199/c-T00/99). Figure 4 shows a photo of the sensor and Table 1 presents its basic parameters. The most important features of the sensor are: remote control of sensor efficiency, automatic correction of thermal fluctuations of a background, automatic control of transmission of input window of optical system, remote identification of a sensor number, programmable operation range, and compatibility with alarm control units of any types. The sensor has filters eliminating solar radiation, optical system protected against environmental factors (rain, humidity) and automatic controls of proper operation of the sensor after alarm system switching (socalled anti-masking). 


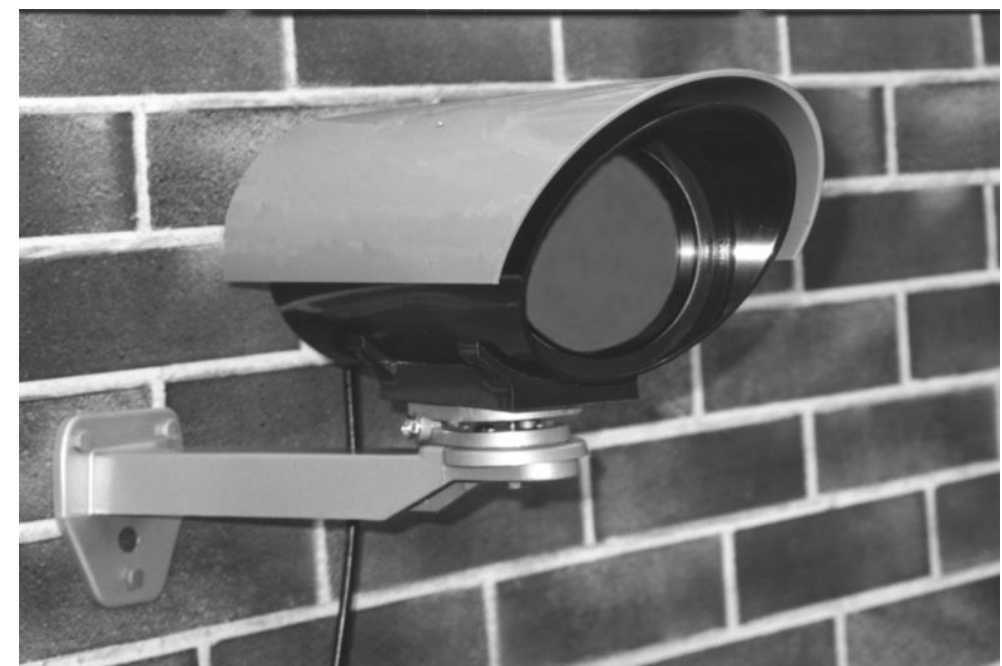

Fig. 4. Long-range passive IR sensor elaborated in the Military University of Technology

Electrical parameters

\begin{tabular}{|ll|ll|}
\hline Supply voltage $\ldots . .$. & 10.5 to $30 \mathrm{~V}$ DC & Time of alarm maintenance........ & 0.5 to $2 \mathrm{~s}$ \\
\hline Supply current ...... & $25 \mathrm{~mA}$ & Time of selftest ......................... & $0.3 \mathrm{~s}$ \\
\hline Transmitter output . & $2 \mathrm{~A}, 30 \mathrm{~V}$ DC & Sensitivity regulation ................. & 10 to $100 \%$ \\
\hline Transistor output .... & NPN(open collector) & Range of operation temperature ,. & -40 to $70 \mathrm{O}$ \\
\hline Digital output ....... & RS 485 & & \\
\hline
\end{tabular}

\section{Conclusions}

Passive radiation sensors used for detection of people crossing optical barrier have optimal range of operation in long-wavelength detection band ( $8-12 \mu \mathrm{m})$. Longwavelength detection band is chosen also due to significantly lower intensity of reflected solar radiation in this spectral window comparing to the 3-5 $\mu \mathrm{m}$ spectral band. Because of low temperature contrast of the object and high probability of false alarms, resulting from fluctuation of background radiation, it is necessary to use twoelement pyroelectric detector operatingin a differential system. Application of optical filters cutting off radiation of wavelength shorter than $8 \mu \mathrm{m}$ significantly decreases the level of false alarms.

\section{References}

1. H. Madura et al., "Performance and testing of optical system and set-up for infrared radiation detection. Performance of (testing elements) "tests" simulating target. Investigation of a system for real objects. Report of the 5-th stage of grant No. 148-35/C50/94, Warsaw, 1995.

2. Z. Sikorski, "Elaboration of theoretical model of optoelectronic thermodetection head used in passive systems for target detection and identification systems and design of a set-up for complex investigations of thermodetection heads". Reports on performance of research grant of the Polish Committee for Scientific Research No. T00A01810, 1997.

3. H. Madura et al., "Review of applicability of passive IR sensors in object protection systems. Analysis of passive IR sensors operating within the range up to $30 \mathrm{~m}$. Application of the analysis results for assumptions of a device operating within the range longer than $100 \mathrm{~m}$. Report from the research work, WZE, Zielonka/Warsaw, 1998

4. H. Madura et al., Analysis and elaboration of IR detection systems applied in object protection. Report from work No. 103/WAT/98.

5. H. Madura, Modelling and testing of IR detection devices. Postdoctoral lecturing qualification, Warsaw, 1998 